\title{
CAMBIOS EN LAS FORMAS Y LUGARES DE CONSUMO EN MADRID
}

\author{
POR \\ EMILIA GARCÍA ESCALONA
}

Lo que se intenta en este trabajo es una aproximación a las nuevas formas de consumo, y cómo estas han modificado las pautas espaciales en Madrid en los años noventa, en atención a la transformación del espacio urbano, a los cambios sociales, y a la incorporación de formas de consumo derivadas de una economía-mundo.

El consumo es hoy abordado por las ciencias sociales desde muy diferentes ópticas ante las nuevas formas de acceso a los bienes, aunque el concepto de necesidad es diferente en épocas, personas y medios de satisfacer lo que se precisa. En las últimas décadas nos situamos en una sociedad de consumo, donde la adquisición de un producto o de un servicio está cada vez menos en relación con la necesidad y más, como señala Metton, A. (1989) con el placer, la moda y diferentes estilos sociales. Las sociedades urbanas evolucionadas, en las cuales también están presentes las necesidades más básicas, han desarrollado una «consumer culture» que es retratada como originaria de los urbanitas de clasemedia educados por las imágenes de las mercancías difundidas por la publicidad capitalista (Thrift. N. and Glennie, P. 1993). De tal modo que afecta al individuo como indica Langman L.(1992) ya que la vida diaria ha sido transformada en una extensión del capitalismo consumista y la persona se convierte en un consumidor o un espectador en razón a la valoración del significado, simbolismo y afectividad del bien, ya que la ideología consumista promete buena vida, bienestar y autoestima. Pos- 
teriormente Atkins, P. (1998, pp. 213) señala como los consumidores postmodernos pueden elegir el lugar y el tiempo, que los estilos de vida tienen gran flexibilidad y variedad, y que los avances en las comunicaciones pueden variar los consumos. Cuando el consumo salió de la crisis, por el lado de un individualismo hedonista centrado fundamentalmente en los objetos de consumo (Alonso, L. E. y Conde, F., 1994). Esta inmersión en el consumo se pone de manifiesto igualmente en avisos, como el que lanzó la CECU (Confederación de Consumidores y Usuarios) en la campaña de Navidad de 1995 en España «Tú eres el mejor regalo, que no te consuman». Estas puntualizaciones hay que contemplarlas en un cambio tecnológico que ha llevado a la globalidad, económica, social, y urbana, con las consiguientes reestructuraciones espaciales, del trabajo y de los mercados, y por supuesto del consumo. En relación a España podemos recoger lo que afirma Goula, J. (1997) en relación a la mejora del poder adquisitivo, creación de empleo, de nuevos hogares familiares, bajada de los tipos de interés y aspectos psicológicos.

«Ahora lo que se produce en todo el mundo está aquí y es difícil saber qué es lo propio» como afirma Néstor García al explicar las diferencias entre internacionalización y globalización ante los conflictos multiculturales de la globalización. O cuando socialmente la tarjeta de crédito, ha venido a sustituir al «apúntamelo en la cuenta», «lo pago a fin de semana» y al «se fía mañana». La sociedad española ha pasado en menos de cuarenta años, de la autarquía por motivos políticos, a la internacionalización; comprábamos fuera aquellos productos «modernos», por ejemplo ropa de tergal, asistíamos al cine y al teatro fuera de las fronteras para ver «lo prohibido», y en los noventa nos incorporamos plenamente al consumo global. De tal modo que ya no es tan extraño que El Corte Inglés, con implantación en las mayores ciudades del país, líder absoluto de los grandes almacenes y una de las primeras empresas del país, dedique unas semanas a organizar una feria de productos orientales (en 1996 a China, o en el año 2000 al Sudeste Asiático) lo más significativo es que la mayoría de la oferta no es novedad ya que su consumo por precio y tipo de producto ha llegado a cualquier punto del país a través de las denominadas tiendas de cien (tiendas que ofrecen todas las mercancías a un precio fijo, 100, 200, 500 o 1.000 pesetas). 


\section{El dominio urbano en la Comunidad de Madrid.}

La Comunidad Autónoma de Madrid se estableció en 1983 sobre la base territorial de la provincia homónima, con una extensión superficial de $8027,9 \mathrm{~km}^{2}$, una base territorial que la posiciona en el sexto lugar, de menor a mayor en la actual división en Comunidades Autónomas, sin embargo en la misma reside el 12,67\% de la población de España en 1996. Administrativamente está configurada en 179 municipios, 28 de los cuales se integran en el Área Metropolitana Madrileña, que supone 1941,9 km² de los cuales 605,8 constituyen el municipio de la capital. La distribución de los cinco millones de habitantes de la Comunidad, viene marcada por la concentración en el Area Metropolitana (90,42\% en 1999), singularmente en el municipio de Madrid que cuenta con el 55,77\% de la población, y de siete municipios con más de 100.000 habitantes y seis con más de 50.000 hab. y menos de 100.000, en 1996.

La evolución de los espacios, la población, y los lugares y pautas de consumo, no son ajenos al carácter singular de Madrid por su capitalidad. La atracción de población a Madrid y el consiguiente crecimiento natural, desde mitad del siglo hasta la década de los setenta, provocó en primer lugar un consumo de espacio urbano, sin estar totalmente rellenado el término municipal de la capital, dio lugar a saltos a otros municipios periféricos, conformándose un modelo metropolitano que ha sido acompañado por una transformación económica, y así en 1999 el 73,44\% del empleo en la Comunidad se situaba en el sector servicios a la vez que es el segundo foco industrial del país, tras Cataluña. Derivado de la plasmación de la aglomeración se ha producido una profundización de la segregación social en el espacio con contrastes centro-periferia y N-S o mejor NW-SE; así mientras que el tamaño de los hogares en la Comunidad en 1996 era de 3,1 personas, en Madrid municipio y en los municipios extremos de la comunidad, mal conectados a la red de carreteras y envejecidos no alcanzaba los tres miembros, en la corona metropolitana aumentaba y sensiblemente en el oeste de la misma. Sin embargo hemos de recordar que en relación al consumo no se pueden equiparar hogares y personas, ya que hay bienes que pueden ser simultaneados por varias personas y otros que son privativos del hogar, sea éste de uno o múltiples miembros. De tal modo que el «consumo privado de bienes y servicios, se estima que ha aumentado alrededor del $2,3-2,4 \%$, con la particularidad de que los gastos en servicios (ocio principalmente ), han crecido entre el 3,5 y el $4 \%$ en tér- 
minos reales» (CCCE 1997, pp. 29) Sin duda el motor de estos cambios ha sido el hecho de la capitalidad, la población ha ocupado espacios, cada vez menos centrados en Madrid municipio, movimiento que siguieron los establecimientos industriales y las empresas, diversificando los lugares de residencia, trabajo y consumo.

Efectivamente ese mayor consumo de espacio se ha producido por las transformaciones socioeconómicas: una población que cuenta con el $15,79 \%$ de los vehículos (turismos) del país, en la que se ha modificado la composición por edad, pasando de joven en los años setenta (un 7,16\% de más de 65 años y 28,23 menores de 15 años) al progresivo envejecimiento (en 1996 un 13,4\% de mayores de 65 y un 15,9\% de menores de 15 años), con crecimiento empresarial y de numero de empleos que se tradujo en la riqueza generada, aumento de los niveles de renta, y elevación del consumo, como afirma Yagüe, M. J. (1998, pp.222) «el ciudadano medio del AMM tiene una renta y un gasto per cápita superior al ciudadano español». En definitiva se configuró un mercado urbano ${ }^{1}$ de amplia base territorial y poblacional que reforzó la tendencia de la economía de los servicios, a lo que hay que añadir la incorporación a la Unión Europea, el fuerte aumento de las inversión extranjera en el país, siendo Madrid uno de los principales focos. Todo ello ha llevado a que Madrid figure en las listas de las ciudades globales de segundo rango. Así lo confirma Estébanez, J.(1994) al analizar los rasgos de las ciudades globales y ratificarlos en nuestra ciudad «desde la década de los ochenta se esta formando en Madrid una ciudad global que extiende su influencia directa por todo su espacio comunitario... Madrid es un centro neurálgico del nuevo sistema mundial, impulsor de los efectos innovadores del nuevo orden económico en toda España». Cuando Precedo, A. (1996) aplica el índice de terciarización postindustrial de la economía urbana afirma «lo más destacable es la preeminencia de Madrid como centro español del terciario postindustrial». La importancia de Madrid en términos de redes de transporte, de actividades empresariales de muy diverso orden y de nudo principal en el dispositivo redistribuidor de mercancías de España lleva a García Delgado J. L. (1999, pp.18) a poner de manifiesto la grandeza de la economía madrileña.

${ }^{1}$ Eurostat (1994), examinando la distribución regional de los establecimientos minoristas españoles señala «el predominio de cuatro regiones: Cataluña $(18,8 \%)$, Andalucía $(16,5 \%)$, el área metropolitana de Madrid $(11,6)$ ๆ Valencia $(11 \%) »$. 


\section{Transformaciones en las formas y en los lugares}

Si nos encontramos ante un espacio urbano evolucionado, con una sociedad que ha cambiado sus valores en relación a la familia, que por el desarrollo económico ha pasado de la «necesidad» ${ }^{2}$, que está inmersa en las nuevas tecnologías y en la globalidad, nos planteamos cómo todo ello ha incidido en las pautas y espacios de consumo y si hemos alcanzado la cultura del consumo. La evidencia señala que sí, con sólo pasear por las calles de Madrid, desde el uso del teléfono móvil, nuevos establecimientos comerciales y de servicios, incorporación a las cadenas mundiales de bancos, empresas, comidas... pero ¿cómo aproximarnos?

Para ello hemos seleccionado algunas actividades de consumo, escasas en el contexto pero creemos que podrían resultar significativas. En razón a que, si bien la ciudad no ha sido extraña a fenómenos de grandes proyectos urbanísticos, fenómenos de renovación y de implantación de nuevas centralidades, baste con recordar el complejo AZCA o El Campo de la Naciones, observamos que en la década de los noventa destaca singularmente la implantación de esa cultura consumista y la incorporación a la globalidad no ya a través de innovaciones, bancos y empresas, que pudieran «afectar» a determinados grupos, sino a través del uso en la vida cotidiana de los madrileños. Estas tres actividades son comercio, restauración y cine que enlazan con la más profunda tradición humana, la necesidad de alimento, de aprovisionamiento y de algo «alegre». Además entre las cuatro principales formas de innovación que el consumo ofrece ahora (Thrift, N. and Olds, K. 1996) tres están relacionadas con la actividad comercial, y en el índice de terciariación postindustrial (Precedo, A. 1996) que señalábamos anteriormente se incluyeron: grandes superficies, centros comerciales, grandes almacenes e hipermercados. Respecto a las otras dos variables parecía evidenciarse: un incremento en nuestra ciudad de los establecimientos de restauración, su asociación espacial a la actividad comercial, y el cine que dentro del ocio podía ser considerado «clásico», se mostraba «postmoderno» en su asociación a las otras dos varia-

${ }^{2}$ Como se deduce de la elevación del número de establecimientos minoristas, cierres en los comercios tradicionales de alimentación, y del gran aumento de la categoría de «diversos» (más del 30\%) que engloba a gran variedad de productos desde joyas, juguetes y artículos deportivos a los aparatos ópticos y médicos. «Esta tendencia refleja la diferenciación y la sofisticación de los gustos de los consumidores españoles» (Eurostat, 1994). 
bles y a sus pautas de localización ya que «los nuevos centros comerciales pretenden atender una demanda más ligada al ocio que a la necesidad a la hora de satisfacer su deseo de consumo y que no tiene en los precios su única y primaria explicación» (Carreras, C. 1995, pp. 74) y teniendo en cuenta que ya se ha asociado «el centro comercial es uno de los elementos de la imaginería postmoderna... donde la familia postmoderna cambia los lugares tradicionales de ocio para reinscribir una antigua noción de recreación: el mercado, hoy día travestido en "malls"», como apunta Ferreira, R. (1996, pp. 29).

\section{La actividad comercial}

La Comunidad Autónoma de Madrid presentaba una situación comercial en transformación al inicio de esta década que estudiamos en un trabajo sobre centros comerciales (Bustos, D. y García, E., 1990). La perspectiva actual parece confirmar las tendencias que se manifestaban al inicio de los noventa: variedad en los canales y forma de comercialización, adaptación a los variados consumos, como señala Casares, J. (1999, pp. 501) «Todas las formas comerciales presentan su «cesta de características» contribuyendo a la creciente segmentación del mercado». Esta situación se verá sin duda afectada por el fin de la moratoria legal para que el comercio (pequeño y mediano) se adapte a los cambios, prevista para el año 2001, y si la situación económica es de bonanza ya que la Encuesta Municipal de Consumo de 1999 señala como posible clave a la preferencia mayoritaria (56\%) por las tiendas pequeñas la disponibilidad de renta « a medida que desciende el nivel de ingresos, es mayor la preferencia por el comercio tradicional» (pp. 26), pero también se ha señalado la «preferencia creciente de los consumidores españoles por la compra en establecimientos de gran superficie» (Alonso, J., Rebollo, A. y Oubiña, J. 2000, pp.139). Mientras que en 1985 se liberalizaron los horarios comerciales en todo el estado español, con lo cual las grandes superficies iniciaron un período de expansión en detrimento del pequeño comercio, ya que ellas facilitaban la amplitud de horarios y el uso de días festivos para compras; en 1993 se limitó por Decreto-Ley, los días de apertura en festivos y las horas semanales (72 horas), pero se permitió que cada Comunidad Autónoma aplicase esta limitación, y la Comunidad de Madrid opto por las restricciones. La nueva Ley de 1996 de Ordenación del Co- 
mercio Minorista, plantea más restricciones a las grandes superficies, por ejemplo impide que los hipermercados (locomotoras de muchos centros comerciales) limiten la cantidad de productos que desee un comprador y que practiquen la venta con pérdidas en determinados artículos para atraer clientes. Igualmente la apertura en días festivos ha implicado problemas, así en la Comunidad de Madrid, 67 municipios se beneficiaron en 1996, al acogerse al Decreto de Zona de Gran Afluencia Turística, pero no así otros como San Lorenzo del Escorial, Aranjuez, Chinchón, o Alcalá de Henares, debido a que la adscripción se hacía por petición de los ayuntamientos y estos municipios dieron por supuesto que eran turísticos. Por otra parte, el comercio pequeño y tradicional puede verse afectado por la también reciente Ley de Arrendamientos Urbanos, que obliga a actualizar el precio de los alquileres de renta antigua en un período de años variable y por la bonificación impositiva si el inmueble esta localizado en el distrito Centro madrileño.

A) La concentración. El panorama presente traduce una tendencia a la concentración de la actividad comercial: por las transformaciones internas en el propio sector, procesos de absorciones y fusiones, cooperación entre empresas, adaptación «a las nuevas necesidades de un mercado cada vez más segmentado» Melle, M. (1999, pp.330); por la asociación de compras a ocio, áreas de actividad laboral y ubicación de la vivienda; por la pluralidad de tiempos y lugares de consumo, y así se manifiesta en el espacio regional:

- En el centro del municipio capital ${ }^{3}$, encontramos confirmación de áreas especializadas, singularidad y agrupamiento de la diversidad asociándose a zonas de intensa actividad laboral, donde el comercio es para los residentes, para las personas que trabajan en esos espacios y no residen en los mismos y para aquellos que van al centro a buscar «lo especial» $\mathrm{o}$ «la oferta variada».

- En los distritos periféricos, aparece una disminución de la dispersión y concentración en ejes y focos que actúan como «centros» y que a otras escalas plantean los valores de centralidad, accesibilidad, con-

\footnotetext{
${ }^{3}$ La división territorial vigente desde 1988 del municipio de Madrid en 21 distritos, los siete primeros (Centro, Arganzuela, Retiro, Salamanca, Chamartín, Tetuán y Chamberí) en su mayor parte delimitados por la M-30, constituyen el espacio central, denominado frecuentemente la Almendra madrileña.
} 
centración y diversidad; así en Madrid se mantiene y están sustituyendo los locales hacia consumos especializados, por señalar algunos ejemplos citaremos, calles como Marcelo Usera (Distrito de Usera), el tramo de la calle de Alcalá desde la M-30 hasta el cruce con la calle de Arturo Soria (D. de Ciudad Lineal), la avenida de la Albufera en el de Puente de Vallecas, o General Ricardos en el de Carabanchel.

- En las coronas metropolitanas ${ }^{4}$ la tendencia a la concentración se manifiesta, por la implantación de nuevos centros comerciales con respuestas también de los pequeños comerciantes que se asocian de forma diversa, cadenas de pequeños supermercados, regalos de cheques de gasolina, como por ejemplo en Leganés o actividades de ocio como se hizo en una campaña de Navidad en Aranjuez.

B) Los centros comerciales. Esta tendencia a la concentración tiene otra forma de expresión que podríamos denominar «todos quieren ser un centro comercial», por la proliferación de centros comerciales y porque estos se ajustan más a los nuevos modelos de consumo que unen compra rutinaria, más o menos esporádica, servicios y ocio:

- Los tradicionales mercados de abastos dedicados a la alimentación, en donde la cultura del carrito impuesta por la gama de autoservicio, se traduce a otro tipo de carrito que el ama de casa tradicional lleva desde su hogar, y que han pasado a denominarse centros comerciales de barrio. Aunque en la mayor parte de ellos se mantienen unos hábitos y usos «populares», pero han introducido mejoras y a veces un nuevo edificio, dotado de escaleras mecánicas y más acorde con una imagen de modernidad, como es el caso en Madrid del ahora denominado Centro Comercial Las Ventas, que ha sustituido al mercado de Ventas, cambiando la ubicación y edificación, y presenta una nueva imagen más en consonancia con las transformaciones de esta zona, límite del municipio a mitad de siglo y hoy en contacto con el espacio central delimitado por la M-30.

${ }^{4}$ Teniendo en cuenta la «elasticidad» del espacio urbano madrileño, en ocasiones hay que hablar de coronas metropolitanas, ya que los municipios incluidos en el Censo de 1991 que configuran el Área Metropolitana, no son exclusivamente representativos de algunos fenómenos; en el caso concreto de la actividad comercial podemos poner el ejemplo del C.C. «Los Valles» (con hipermercado Pryca) a $40 \mathrm{~km}$ por la autovía de la Coruña, y del Zoco que se ubican en el termino municipal de Collado Villalba (26.356 h. en 1991 y 36.950 en 1996). 
Podemos sugerir que esa «cultura del carrito» sea la expresión del paso de lo «necesario»: implica un pago al final, no producto a producto; refleja las transformaciones tecnológicas en el hogar; expresa una disponibilidad de renta; induce a incluir en el «carro» productos no previstos; permite la atracción de la mercancía autoservida frente a la petición tras el mostrador. Esta «facilidad» es practicada por pequeñas tiendas, «super», «hiper»y «boutiques» de lujo, y se enfrenta en la pluralidad actual, al cierre tras cristales blindados de mercancías y dependientes, acceso controlado o servicios de seguridad diversos, que en razón a la delincuencia se ven obligados a instalar, estancos, farmacias, joyerías... etc.. El «carro» de la compra es quizás, hoy la mejor cualificación del sector: su no utilización por aquellos que pueden pagar un servicio para que el producto les llegue a domicilio; a su manejo por los que pudiendo asumir el coste de este servicio, optan por transportar su compra en razón al tiempo y al capricho, en una sociedad de consumo que lucha por vencer no sólo el espacio sino también el tiempo y que se permite «querer algo y ahora». El carro se ha adaptado a los bebes, a los niños, para comodidad de los padres, o reduce su tamaño para iniciar a los «pequeños» al consumo; se ha acoplado a las sillas de ruedas de los inválidos; y ha ajustado su tamaño a la mercancía. La diversidad del mercado y de los consumidores podría contemplarse a través del papel donde se envuelve lo necesario o lo superfluo; la bolsa de plástico o la ausencia de la misma en cadenas «baratas»; la bolsa o caja de «diseño» con un logotipo y el nombre de «ciertas ciudades», correspondiente a establecimientos selectivos por su precio y ubicación en calles, y centros comerciales que no disponen de carro (en Madrid, ABC, Jardín de Serrano, Galería del Prado, Moda Shopping); a los dos modelos de carro de Ikea donde también el comprador se puede limitar a la bolsa.

- Los hipermercados, las grandes cadenas que operan en Madrid en su publicidad señalan el número de centros, la dirección o incluyen esquemas de localización, y que se ubican en un centro comercial con diversidad de comercio y servicios, o bien establecen en sus edificios una galería de entrada, donde se instalan tiendas y servicios complementarios, intentando alejarse de la imagen rutinaria de la compra de alimentos, y perdiendo parte de su inicial papel de locomotora de los centros comerciales, pero sumando a su imantación los valores del ocio ( cines y deportes). 
- El comercio especializado que se instala en calles de «status» ${ }^{5}$ o en los nuevos centros comerciales (tipo Galería del Prado, Jardín de Serrano, ABC, Moda Shopping) donde el carrito no presenta preeminencia, ya que no cuentan con la locomotora del hipermercado, aunque los menos céntricos (tipo Ärturo Soria-Plaza, C. C. La Moraleja) tienen supermercados selectivos en cuanto precios y productos. Podemos contemplar dos ejemplos de multiplicidad de lugares pero de importancia de la localización: las tiendas Roberto Verino, sitas en la calle de Serrano y Claudio Coello, igualmente en los C.C. de Arturo Soria, Moraleja y Moda Shopping, y en los centros El Corte Inglés; y el supermercado Sánchez Romero, cuyo «carrito» es significativamente de menor tamaño, y que inició su expansión en la calle Carlos Maurras (esquina al paseo de La Castellana) y manifiesta su vocación de localización, ya que se ubica al norte de la línea oeste-este (José Abascal-María de Molina) en zonas percibidas por los madrileños como de «buena calidad» (Puerta de Hierro, Sor Ángela de la Cruz, final de Corazón de María) y en los centros comerciales de Mirasierra, la Moraleja, o Arturo Soria.

- Los polígonos, espacios delimitados y calificados para albergar usos de suelo industrial, por iniciativa pública o privada, en razón a los precios del suelo en las periferias urbanas; conceptualizados más reciente de parques empresariales e incluso tecnológicos y ¿porqué no serán denominados a futuro, parques o polígonos comerciales? dado que las naves de algunos se han convertido en contenedores de variedad de «tiendas», por ejemplo el Polígono Industrial Norte en San Sebastián de los Reyes o el Parque Tecnológico Európolis en las Rozas. En ambos casos encontramos un establecimiento, Main Out Let, especializado, que se incluiría en la clasificación de Casares, J. (1999, pp. 496) entre las «Grandes superficies "emergentes"» («factory outlets», parques de fabricantes), pero a este se han unido otras superficies especializadas y hoy constituyen también un entorno comercial que dado el tamaño de las naves es recomendable realizar en automóvil, que por otra parte es el modo de acceso a estos polígonos y el emblema de los espacios suburbanos. Esta forma ha emergido ya en C.C.Parque Alcorcón, un gran polígono comercial, situado en la carretera de Extremadura y al oeste del municipio, donde se ha instalado entre otros, Ikea, Alcampo, Leroy

${ }^{5}$ En diciembre de 1999 un articulo del diario El País se titulaba «Preciados la calle más cara de España» señalando las 16.500 pesetas por metro cuadrado de alquiler mensual de locales en la misma, seguida por las 14.500 pesetas del precio en la calle de Serrano.

$$
-82-
$$


Merlin, Porcelanosa; y en el denominado Megapark (N-1, San Sebastián de los Reyes) donde se localiza Ikea y Media Martkt.

- También, Madrid ha incorporado a su espacio comercial las tiendas de fabricantes, basadas en la cultura del consumo ya que sus artículos de primeras marcas proceden de los excedentes de temporada, ofertados en estos enclaves a un precio como mínimo de un $30 \%$ todo el año, y tras el éxito en la carretera de A Coruña (Las Rozas), Factory, abrió en noviembre de 1999 otro complejo de 57 tiendas en la carretera de Andalucía (Getafe).

- Por otra parte el ejemplo de la salida número 19 de la carretera de La Coruña, plantea cuestionarnos una futura forma de «centro comercial», en espacios amplios, a escala de automóvil (por distancia y no viabilidad peatonal) pero donde se «... agrupan...» hipermercado (Pryca), gran superficie especializada (Leroy Merlin), tiendas de fábrica (Factory) y centro de ocio (Heron City).

- Incluso los pequeños comerciantes, mediante el asociacionismo, pretenden dar esa imagen de concentración comercial, agrupando la publicidad o bien como ha sucedido en Madrid, en el corazón del casco histórico, donde la calle peatonal de Preciados, se ha cubierto con arcos de los que penden adornos de colores, contribuyendo a una imagen de galería por otra parte muy festiva.

C) La masificación. Otra manifestación de la nueva actividad comercial es la masificación y la estandarización:

- Las familias compran para mayor número de días, se compra por cajas determinados productos y aparece la imagen de los carritos convertidos en «camiones».

- La imagen de la misma tienda, lo que se llamó la hiltonización o sheratonización: los Body-shop; las tiendas Natura con su antinatural oso disecado en la entrada que ya no nos sorprende al encontrarlo en distintos centros; la cadena española Zara; los almacenes ingleses Marks \& Spencer; la cadena americana de juguetes Toys «R» Us ... Contemplados espacialmente en Madrid parecen tener el don de la ubicuidad, calles del centro de prestigio y populares (Zara está en el ABC de Serrano y en la calle Carretas, entre otros puntos); Marks \& Spencer en la calle Serrano, en la Vaguada, en el C.C. Oeste de Majadahonda; la danesa Bang \& Olufsen, en C.C. La Moraleja, en el C.C. Burgo de la Rozas, en C.C. Arturo Soria, en el $\mathrm{ABC}$, pero también se ubica aislada en calles (Comandante Zo- 
rita o Alberto Aguilera); Toys «R»Us «tiene» 26 tiendas en España, cuatro de ellas en C. de Madrid (en C.C. de Alcorcon, C.C. Parque Corredor, C.C. Río Norte y otra independiente en Majadahonda). A la difusión de la misma imagen han contribuido las franquicias que «han encontrado en Madrid un lugar particularmente propicio para su expansión» ya que el 35,5\% de los franquiciadores tienen su sede en Madrid y el 14,9\% de los establecimientos franquiciados en España (Méndez, R. y Ondátegui, J. 1999, pp. 155).

- Los mismos productos, incluso los de lujo y elitistas, ya que las grandes firmas, por ejemplo de costura, comercializan perfumes o complementos que pueden ser adquiridos por mayor número de consumidores, aunque sólo sea ocasionalmente, así podemos comprar un aroma del francés Cartier o del español Loewe en multitud de perfumerías; igualmente no parece sorprender que los hipermercados Alcampo, en su publicidad, ofrecían un «Conjunto de Golf Profesional Spalding», aunque no es una actividad masiva en nuestro país y por otra parte teniendo en consideración su localización en Madrid - Vaguada, Pío XII, Moratalaz, Vallecas (en la capital) - y Alcalá de Henares, Torrejón de Ardoz, Getafe, Alcorcón, Leganés (en la corona metropolitana) coincide precisamente con clases sociales medio-altas ${ }^{6}$.

D) La pluralidad. La especialización no es ajena a las nuevas fórmulas comerciales y presenta un carácter muy diverso:

- Cadenas de «hard discounts», «not frills», marcas blancas, dispersas en la mancha urbana, básicamente de alimentación envasada, que se instalan en locales pequeños, con escaso complemento a la compra (por ejemplo no dan bolsas para llevar los productos), y escaso número de cajas de abono, como las cadenas Día, Superdescuento, Lidl, etc.

- Grandes superficies especializadas («category killers») en bricolage, hogar, jardinería, muebles etc., asociadas a las salidas por carretera y ubicadas en las periferias de la ciudad central, con concentración pero no exclusión en determinados ejes. Por ejemplo Texas (en Alcobendas y las Rozas); Leroy Merlin en la autovía de La Coruña; Media

${ }^{6}$ Distritos y municipios, que fueron ocupados por promociones de viviendas ante la inmigración masiva, incluso estigmatizados, asociados a la instalación de la industria, y reivindicados «El Sur también existe» desde diversas instancias políticas. 
Mart «todo lo que puedas enchufar» en San Sebastián de los Reyes; El Corte Inglés va a abrir un gran centro de bricolage en Valdemoro donde por otra parte tiene ubicados sus depósitos de mercancías; centros de jardinería en las carreteras de Colmenar o La Coruña; muebles en los ejes del Sur, que ya parecen notar la reducción de los días festivos en los que se podía comprar, como ha manifestado en los medios de comunicación la Asociación de Comerciantes de Mobiliario de Extrarradio de Madrid y Provincia.

- Especialización para determinados grupos sociales, y en este caso la localización se produce básicamente en el espacio centro de la ciudad. Así en los siete distritos centrales de Madrid, encontramos por señalar algunos ejemplos, tiendas dedicadas a: tallas grandes (G. C., Amplisimo, Superwoman); niños (Jacadi Paris «La marca de la Infancia», que señala tener 450 tiendas en el mundo y cuenta con tres establecimientos en nuestro espacio central y otro en el C. C. La Moraleja); tribus urbanas con particular ubicación en el casco histórico (Marihuana, Kaos, Triburbana) o para los que se adornan con tatuajes y piercing; para aprendices de mercenarios (Soldiers); para gourmets, Black Pearl o Gold Fish, los locales del caviar iraní, o El Rincón del Cava que provee de 25 tipos diferentes de cava. Parte de los tradicionales herbolarios han sido sustituidos por la moda ecológica que nos lleva en Madrid del Autoservicio Ecológico, Bioladen, Central Vegetariana o Maurice Messegué. El «amor a los animales» o la moda de la mascota han llevado a la proliferación de clínicas, peluquerías, tiendas, pasillo de estanterías en los super o hiper, catálogo específico (El Corte Inglés edita 12 páginas, en las que se incluye abrigos, impermeables, jersey bordado y delicatesen en alimentación); este «amor» puede llevar incluso a la extrema especialización, como se deduce de la recién abierta tienda de los loros «Europ@rrot» en la calle de $\mathrm{Mu}$ rillo, junto a la plaza de Olavide.

Es la prosperidad del comercio, en el centro de la ciudad, cuando parte del no rentable ha desaparecido y los comerciantes se han adaptado a una clientela más exigente, y donde también aparecen las franquicias y los grandes grupos, como los especializados en ocio. Igualmente podemos señalar en Madrid, la instalación en 1993 de la francesa FNAC, $7000 \mathrm{~m}^{2}$ en la plaza de Callao, la inglesa Virgin en la Gran Vía y en el ABC de Serrano, la respuesta del español Corte Inglés, dedicando locales exclusivos, fuera de sus grandes inmuebles, a vídeos, discos libros y revistas; o la expansión de Crisol, nueve establecimientos en espacios centrales del municipio ca- 
pital. Así parece que responde el espacio central, con la especialización, que aparte de ofrecer tiendas a los que en él trabajan, estas refuerzan su terciarización, y mantiene sus cualidades de «ciudad» para aquellos consumidores que desde otras partes de la aglomeración disponen de más tiempo y dan más importancia a la calidad del producto derivada de una atención personal del especialista. Este rasgo parece reforzarse ya que fue característica de la urbe, que desarrolló calles asociadas a una actividad concreta, de las que en Madrid aún permanecen en el nombre y en la actividad (Libreros, Platerías), frente al mundo rural donde en el bazar, el colmado o en «la tienda» se encontraba «todo», y que hoy es sustituido en la corona metropolitana por los centros comerciales. En éstos también se puede encontrar de todo ya que su concepción recuerda la idea de una ciudad ideal (Ferreira, R. 1996), e imitan ese carácter de la ciudad mediante la creación de «Calles de la Moda» como sucede en Madrid en la Vaguada o en Parquesur.

E) La dificultad espacial. Ante el cambiante papel de los lugares de consumo, la multiplicación de los focos, la concentración-especialización, la combinación de ocio, servicios y comercio, centros comerciales de compleja tipología (céntricos y periféricos), valores de marca y del glamour, hemos optado en nuestros ejemplos por seleccionar los nombres originales, que consideramos expresivos de la transterritorialidad que se acompaña en las ciencias sociales por el énfasis en la localidad, y se traduce en el espacio urbano en áreas de diferentes estigmas y estatus, y teniendo en cuenta que las implicaciones en las nuevas culturas, cambian las prácticas espaciales y las representaciones, así en el análisis de los hipermercados de la Comunidad de Madrid, se señala la importancia de la imagen ya que la localización y el tamaño de la tienda son variables muy importantes en la elección del establecimiento, pero no suficientemente explicativas (Ballesteros, C. 1999). Por todo ello hemos optado por aproximarnos a través de algunos ejemplos de publicidad, sabiendo que en los análisis de consumo se señala que el actual usuario está mejor informado. Efectivamente, desde todos los medios de comunicación a través de la publicidad podemos obtener esa información y además la especialización se manifiesta en revistas para cualquier línea de consumo. No hay más que acercarse a un puesto de periódicos, guías de ocio, compras, ordenadores, informática, automóviles etc. que pueden llevar a la necesidad de estudiar para estar informados, o si nos parásemos ¿que jabón de lavadora elegir 
ante la proliferación de marcas?... y ¿porqué no utilizar la publicidad? cuando en la conclusión al trabajo «Geography and the cultural politics of advertising» (Jackons, P. and Taylor, J. 1996) señalan la negligencia de la investigación geográfica en la publicidad... Efectivamente, las ciudades se venden y los diferentes espacios urbanos también, la publicidad se inseta en vallas, en azoteas y tejados, o en los medios de transporte. Pero en los nuevos centros comerciales se valora el entorno, la imagen, se han convertido en la nueva calle, donde se puede pasear, sentarse en una terraza «al aire», ver una película, comer, jugar (niños y adultos), practicar deportes, ir a la peluquería, y por lo tanto intenta presentarse como la continuación de la tradición de los espacios públicos (Shields, R. 1992).

Pues bien, hemos de recordar que:

- El primer centro comercial integrado, con locomotora, un gran almacén (antes Galerías Preciados hoy El Corte Inglés), el hipermercado Alcampo, bolera, mercado, calle de la moda y galería de artesanos, restaurantes, amén de 9 salas de cine. Este centro, incorporó el aire libre a través de claraboyas, árboles y plantas, se abre en Madrid en 1983 (en el distrito periférico de Fuencarral) conocido por La Vaguada porque su emplazamiento dio lugar a una amplia polémica, tiene por nombre oficial «Madrid-2», ¿reflejo de su pretensión de ofrecer «otra ciudad»?

- El complejo abierto en el distrito de Carabanchel se llama C.C. Plaza Aluche (la denominación del barrio). También el «C.C. Arturo Soria-Plaza» en el distrito y calle que recibe el nombre de este urbanista, ¿pretende sustituir la centralidad tradicional de la plaza?

- En los municipios de la corona metropolitana encontramos: Centro Comercial Parque Alcorcón (donde en septiembre de 1996, abrió «Lo que será el primer gran centro del mueble y la decoración en Madrid» según se anunció a toda página en prensa, la sueca IKEA y también se decía «tenemos abiertos 130 centros en 28 países» ${ }^{7}$, y en 1999 inauguro otro en San Sebastián de los Reyes y señalaba su presencia con 149 tiendas en

${ }^{7}$ Desde 1958, los muebles de Ikea se han mundializado desde Älmhut (Suecia), expandiendose a Noruega (1963), Dinamarca (1969), Alemania (1974), Australia (1975), Canadá (1976), Austria (1977), Singapur (1978), Holanda y Suiza (1979), llegan a Las Palmas de Gran Canaria en 1980, Islandia (1981), Francia (1982), Arabia Saudí y Santa Cruz de Tenerife (1983), Bélgica y Kuwait (1984), U.S.A. (1985), Reino Unido (1987), Italia (1989), Polonia y Hungría (1990), Dubaí (1991), Suiza, Eslovaquía, Hong-Kong y Palma de Mallorca en 1992, Taiwan (1994), a Malasía, Finlandia, Barcelona y Alcorcón en 1996, China y Rep. Checa en 1998. 
29 países y cuya estrategia de desarrollo cumple con lo que Soumagne, J. (1999, pp. 106) denomina «fases espaciales sucesivas»; C.C. Fuenlabrada-2, C.C. Getafe-3, o Ciudad Comercial las Lomas (identificación con la urbanización de status medio-alto), Zoco de Pozuelo, Zoco de las Rozas, Zoco de Majadahonda. «Parque Corredor» en Torrejón de Ardoz, ¿en alusión a lo que se denomina el corredor Madrid-Guadalajara o corredor del Henares?, este C.C. se instalo al Norte de la N.II, «al otro lado de Torrejón», rodeado por tierras de cultivo y se anuncia como Parque Comercial y de Ocio, cuenta con su «Paseo del Ocio», su «Calle de la Moda», y «Calle del Comercio», y efectivamente el ocio está bien representado por gimnasio, piscina, área de raqueta, fútbol-sala, centro de diversión familiar, área infantil («Baby West») y un gran karaoke («Megacity»).

- Incorporándose otros nuevos centros, cuyas nominaciones son el eco de... «C.C. La Gran Manzana» ¿Manhattan en Alcobendas?; «El Ferial» ¿acaso en Parla es un centro ocasional?; «La Dehesa» er. Alcalá de Henares ¿una dehesa junto al río Henares?, donde se ubica Decatlon que se anuncia «un 10 en deporte», aunque también podemos encontrar a este especialista en dos centros comerciales de Alcobendas y Alcorcón. El denominado Alcalá-Norte que está en la acera sur de la calle que es un eje urbano oeste-este.

- Denominaciones que los madrileños identifican claramente, por ejemplo:

- «C. C. La Moraleja», situado en el parque empresarial pero separado por la autovía de la urbanización de La Moraleja (área residencial de clase alta en Alcobendas), inaugurado en 1995, con especial cuidado de los elementos interiores, y la instalación de una cúpula de cristal de 32 por $18 \mathrm{~m} 2$ desplazable para lograr el aire libre, ascensores panorámicos, 8 salas de cine, y señala en su publicidad: a $4 \mathrm{~km}$ del Paseo de la Castellana, acceso a la N-I, conectado con M30 y la M-40 y «Sólo lo mejor».

- «C.C. Sexta Avenida», en la carretera de La Coruña, no tenemos en Madrid avenidas numeradas, y recuerda a Nueva York, se anuncia «El nuevo centro de Madrid tiene otro aire» y señala su entorno privilegiado de estatus residencial «El Plantío, La Florida, Aravaca, Pozuelo, Somosaguas y Puerta de Hierro» en este último caso bastante más separado pero en el mismo eje. 
- «C. C. La Esquina del Bernabéu», anexo al estadio de este club de fútbol e hito espacial en la Castellana, que se anuncia «El otro nivel de Madrid».

- «C.C. El Jardín de Serrano», sito en una manzana, que si bien la fachada oeste da a Serrano, el C.C. ocupa los edificios rehabilitados del este y ninguno de sus dos accesos da a la calle de Serrano; pero Healey \& Baker en «Main'95 Streets» la incluyen entre las calles más caras del mundo. En este centro se ha instalado la joyería Bulgari y en la prensa se anuncia: «Allen Edmons Store, los zapatos que usa a diario el presidente de Norteamérica. Hay dos sitios donde usted puede ver unos zapatos así: Los jardines de la Casa Blanca y El Jardín de Serrano».

- «C.C. ABC» localizado en el rehabilitado edificio del periódico del que recibe el nombre, mantiene la parte noble del mismo, decorada en estilo sevillano y habilitada para restaurante de prestigio, sus fachadas y accesos son por la calle de Serrano y Castellana; su localización en el distrito de Salamanca le llevan a consideraciones de estatus, pero la mezcla de tiendas no hace inaccesible por precio a una mayor parte de la población.

- «La Galería del Prado», emplazada en el mismo edificio del Hotel Palace, toma el nombre del vecino museo del Prado y fue el primer centro de élite en espacio central que se abrió en Madrid, tiene 39 tiendas de firma de primera calidad, precios y marcas que contribuyen a su especialización, el primer restaurante madrileño de las estrellas de cine «Planet Hollywood» y a cuya imagen, no cabe duda, también su ubicación, ya que enfrente se sitúa el Hotel Ritz de Madrid, la Iglesia de San Jerónimo donde se casan los reyes y la clase alta, y la Bolsa, y en su misma calle se codea con el Palacio del Congreso de los Diputados y el museo ThyssenBornemisza.

Tal proliferación de centros comerciales en Madrid, en ámbitos bien diferentes de la aglomeración urbana, refleja los nuevos hábitos de consumo (compra, restauración, ocio, servicios); la concentración de las actividades en focos que pueden solucionar las necesidades, la combinación de una incorporación femenina a labores no domésticas y la integración del hombre, junto con el menor número de hijos, al ocio-compra; asimismo refleja la accesibilidad exarcebada por el automóvil, y 
«una seguridad» relativa derivada de la vigilancia por parte de empresas privadas en estos espacios privados-públicos, y puede que la falta de tiempo y la dificultad de «dominar» espacios urbanos amplios.

F) La tradición renovada. El espacio madrileño mantiene algunas formas y enclaves comerciales más o menos «tradicionales», pero muestran su adaptación a los nuevos gustos:

- Una actividad comercial no sedentaria y al aire libre, en días fijos que va: desde El Rastro en el casco histórico, que ha incorporado puestos de «moda», bisutería, para jóvenes, artesanía, discos compactos, a su oferta de cosas usadas, viejas y antiguas; al Rastro de Tetuán de mucha menor entidad en todos los aspectos; y llega hasta los rastrillos de barrios y municipios limítrofes a la capital, que venden productos frescos al igual que ropa, calzado, artesanía, y cuya fama dependen de la ubicación, día de celebración y por lo tanto de su adaptación a lo nuevos tipos de consumidores; un buen ejemplo de adaptación y prestigio de la ubicación, es el mercadillo de Majadahonda que atrae población del sector oeste y del «centro», la forma comercial no es importante si se adapta al consumidor, y en este caso la vocación del mercadillo se acopla a un estilo de vida medio-alto, del «lujo» a lo «pijo», pero a precios más asequibles. Sin olvidar otros mercados especializados, por ejemplo La Plaza Mayor que los domingos «recupera su carácter» con los puestos y los coleccionistas sin puesto, de filatelia, monedas, tarjetas postales, a lo que se ha unido las tarjetas de teléfono, a la vez que en Navidades se instalan casetas tanto para el Belén como para el árbol o el fin de año. En otra plaza cercana, la del Conde de Barajas, los domingos se instalan los pintores que exponen y venden sus cuadros. La actividad comercial no sedentaria se puede buscar en Internet, en donde se insertan páginas de «fleamarkets».

- Otro aspecto del comercio no sedentario, son las ferias, porque parece cierto la frase madrileña de que «en Madrid hay gente para todo». En el espacio central, predominan las que se instalan al aire libre, desde la Gran Feria del Libro que se celebra en el parque del Retiro y que ha incorporado actividades lúdicas, a la más pequeña del Libro Antiguo y de Ocasión (que además tiene un mercado estable en casetas sitas en el espacio central especializado en cultura) y que se instala dos veces al año en el paseo de Recoletos, lugar donde en otros días del año se celebra la Feria de Artesanía, pero casetas de artesanos de diversos 
países, reflejando la mundialización del consumo, se instalan temporalmente en AZCA y Moncloa. A la vez que los madrileños y foráneos han incorporado rápidamente la nueva localización de los recintos feriales (IFEMA) en un distrito periférico norte, que para profesionales y público en general han llevado a que Madrid figure en las listas de ciudades organizadoras de tales eventos (señalamos en particular las ferias del ocio y tiempo libre, anticuarios, o el salón del automóvil, recuperado después de 52 años y en competencia con el de Barcelona). En una Comunidad esencialmente urbana, también aparece «el campo» como una expresión de la incorporación de lo local; y en este sentido podemos citar la Feria Agromadrid que se celebra en Villarejo de Salvanés, y la promoción de productos de denominación de origen «Vinos de Madrid», o «Aceitunas de Campo Real» en numerosos establecimientos.

- Una faceta del comercio que presenta en la actualidad un cierto retroceso, son los grandes almacenes, espacios cerrados y menos variopintos que los centros comerciales, y cuyo futuro depende de la capacidad de adaptación a los nuevos modelos de consumo madrileño. En este sentido el monocultivo ejercido por El Corte Inglés, quien al desaparecer Galerías Preciados y Sepu ha ido comprado sus locales, se adapta a los nuevos tiempos incorporando cafeterías, restaurantes, tiendas del gourmet, boutiques de firmas, supermercados y diversificando edificios por especialidades; y con nuevos establecimientos centrados en hipermercado (Hipercor) y las tiendas Cortty, dos de ellos ubicados en municipios de la corona metropolitana y otros tres en el municipio central. La Comunidad de Madrid es la autonomía que cuenta con mayor número de centros del Corte Inglés, y espacialmente sus establecimientos tienen una gran importancia como focos de atracción y dinamismo de otros comercios y servicios en sus proximidades. Así ocurre con el foco de Preciados, donde recientemente el emblema del Corte Inglés se ve en edificios de la Puerta del Sol; en el área de Goya, en la de Princesa, o en la supermanzana de AZCA. El poder de atracción de esta gran empresa, beneficia a las otras actividades, se ha «comido» a algunos pero no a los que han sabido renovarse ni a los «modernos».

- Tenemos igualmente que destacar por su importancia espacial, el comercio de lujo, que se trasladó desde la Gran Vía al «barrio» de Salamanca. No es novedad el ejemplo de Loewe, aunque en estos últimos años ha ampliado sus dominios en Serrano, abriendo una tienda 
para hombres. Sin embargo, otro mito del lujo, la joyería-relojería Grassy que se localiza en un edificio singular en el inicio de la Gran Vía, donde tiene un museo del reloj, abrió un local en José Ortega y Gasset. La renovación en esta zona, viene marcada por los centros comerciales especializados, y por la cada vez mayor implantación de tiendas de firma mundializada, ocupando locales muy caros, alternándose con oficinas bancarias, anticuarios, salas de subastas, y galerías de arte, amén de restaurantes y los nuevos establecimientos de comida de trabajo. Se trata de una parte del Ensanche con intensa rehabilitación de edificios, cambios en los locales, expulsión de lo que no se adapta a la impronta de la zona, marcas exclusivas, etc. El área del «lujo» se concreta en estos momentos, dentro del distrito de Salamanca, en parte de dos barrios, tiene su limite occidental en el eje de Recoletos-Castellana, al Este la calle de Príncipe de Vergara, al Norte la de Juan Bravo y al Sur la calle de Alcalá.

Dentro de esta zona se habla de Serrano, pero quizás por su mayor difusión, porque dentro del lujo hay mayor diversificación, pues en su mismo tramo conviven grandes almacenes (Mark \& Spencer y el Corte Inglés) y el C.C. ABC, sin olvidar que ahí está Cartier, entre otros; pero parece insinuarse una prolongación del carácter de esta vía, desde su misma confluencia a los tramos próximos de calles perpendiculares. Aquí se instalan los recién llegados, aquellos cuyos locales podemos encontrar en la ciudades globales.

Podemos citar, como ejemplo de máxima concentración, la calle de José Ortega y Gasset, calificada en la guía «Madrid 2000» de «milla de oro», en las plantas bajas de los edificios de sus tres primeras manzanas se sitúan:

- Adolfo Domínguez, el gallego de «la arruga es bella» que se anuncia en toda una página del diario El Mundo - con sólo su nombre- y «Place de la Madeleine. París».

- Helena Benarroch, «la piel española», desafiando a los ecologistas, que abrió su tienda en la más recóndita calle de Zurbano, y ahora se instala también como dice su publicidad «a ambos lados de la Castellana» al igual que se centra en «la pasión por lo accesorio».

- Necesitamos publicidad para hablar de: Gianni Versace, Hermès París, Giorgio Armani, Escada, Rodier, Louis Vuitton, Kenzo, Calvin Klein o Chanel. 
- Otros en sus selectas fachadas, señalan discretamente, su presencia en otros lugares, el lujo global con los nombres: la joyería Pomellato («Milano, París, Tokyo, Osaka, Capri, Pirenne»). Pero también lo hacen los «media goods stores», las tiendas especializadas de los noventa que han devenido familiares en las capitales europeas, como Häagen Dazs (USA) (Healey \& Baker, 1995), en su heladería y salón de té, en esta calle señala que tambien está en («London, París, New York, Milano, Monte Carlo»).

- Queremos destacar la alternancia en estas tres manzanas, de las firmas antes indicadas, con cinco oficinas bancarias, dos restaurantes, otro disenador de joyas (Marjo); y de dos establecimientos «tradicionales» pero adaptados a los nuevos rumbos, unas mantequerías que ofrecen en sus escaparates delicias de productos regionales españoles, una pequeña tienda de reparación de calzado que quizá sobreviva por su escaparate lleno de productos ingleses para cuidado del cuero.

$\mathrm{El}$ «barrio de Salamanca» presenta otro eje que tiende a especializarse en «lo exquisito de lujo», Jorge Juan. Centrado en la rehabilitación de su callejón, que recibió en 1994 el Premio Especial del Ayuntamiento de Madrid por su contribución al medio ambiente, y donde se ubica «El Amparo», uno de los trece restaurantes madrileños incluidos en la guía Michelín, la tienda de ropa de la diseñadora Sybilla, el establecimiento de zapatos de Robert Clergerie, entre otros. En la calle Jorge Juan y en sus proximidades se localizan: «Weekend» (la tienda para vestir los fines de semana) con artículos de Polo Ralph Lauren, Façonnable, Tommy Hilfiger o Tod's; el local que recupera lo clásico británico en decoración y ropa masculina «Hacket» con nueve tiendas en Londres, y otras en París, Tokyo y Osaka; vecinos son «Mulberry», «Scape of Scotland «, el dedicado al arte de la lencería «Meye Meir»; esta oferta se complementa con restauración selecta y se produce en edificios rehabilitados. Como ejemplo, a ese foco se ha sumado «Vinçon», ocupando en el interior de un manzana el edificio de una fabrica de platería y que ofrece en su publicidad «un extenso surtido de objetos de toda clase en general» y en cuya entrada se ha dispuesto un lugar para dejar la mascota atada y con provisión de agua.

- Incluir en este epígrafe el telemercado puede resultar en principio sorprendente, pero examinando la creciente oferta a inicios del año 
2000, parece que estamos ante una nueva forma y lugar de consumo. Algo que cambia el espacio doméstico y convierte la casa en un escaparate (Padovani, E. 1999, pp. 270) o una adaptación de los antaño servicios a domicilio, aunque trasformados por el uso de medios técnicos más rápidos. Las modalidades de la telecompra se anuncian como la sustitución de las dificultades físicas, una manera de solucionar situaciones de aislamiento y de hiperactividad. Su manifestación en Madrid, parece relacionada con los cambios de comportamiento familiar, y del uso de la vivienda (home information revolution). Pero que duda cabe que la palabra «tele» se ha incorporado a diversas modalidades de consumo: desde los servicios de distribución a domicilio y compra por teléfono a través de los catálogos, de las grandes superficies a otras de menor tamaño, de empresas especializadas, como las que se dedican a congelados («Telefrío», «Bofrost», «Eismann»), al «Telemascota» (alimentos para animales de compañía), y al «Televídeo» (entrega y recogida a domicilio de películas); incluida una amplia variedad de servicios de comida, del catering al «just-in-time», inaugurados por la telepizza, a lo que se incorporó también la comida china y más recientemente la japonesa, el tele-burguer, el tele-pollo, y también los acoplados al gusto tradicional: tele-tortilla, telepaella, tele-pescaíto, o tele-lechal, y los que proporcionan bebidas desde la 19 horas a la madrugada, tele-copas, tele-cubatas, o los que facilitan una celebración en casa «Telefiestón» o «Telefelicita». La compra desde casa se completa con la red (www.) que merece un estudio exclusivo, al igual que la efectuada «sin lugar fijo» por la tercera generación de telefonía móvil (multimedia móvil), y también la compra en «Jamás $1 \mathrm{~m}^{2}$ ha dado tanto de sí», la supertienda vía plus que se anunciaba a 31 de diciembre de 1999 con más de 1000 puntos de venta en España. Lo que algunos ven como una expresión del individualismo, de la conquista del espacio y del tiempo, que permitiría una ruptura con lo social, incita a plantearnos: ¿comprarán los seres humanos un bien para su exclusivo disfrute personal, no necesitaremos ir de «tiendas»?, ¿se incorporarán determinadas marcas a la venta o solo a la información en la red?, ¿seremos tan racionales que compremos la mejor oferta, económica, saludable, biológica, «justa»... y prescindamos del «lugar» y de sus connotaciones?, ¿o más bien incorporaremos al consumo más posibilidades, cuando la tecnología nos permita vencer más al espacio y al tiempo?, Mcinstosh, A. (1997, pp. 81) teniendo en cuenta que comprar permanece como un experiencia social, predice para el «leisure retailing» al menos otro milenio. 


\section{La restauración}

La sociedad madrileña se ha incorporado al fenómeno de «comer fuera de casa» con la misma rapidez que ha crecido y se ha especializado el espacio urbano. Incluso en parte, se ha perdido la comida en casa, familiar del domingo, ya que también es día festivo para las mujeres que trabajan fuera de casa y para el «ama de casa». Esta nueva pauta viene asociada a la presencia en cada «barrio» de restaurantes «no caros», y así han proliferado los chinos, las hamburgueserías ${ }^{8}$, y las pizzerías; a estos se añadieron otras modalidades de comida rápida, (Pans, Subway, Aris) que se ubican también en los centros comerciales y en zonas de intensa actividad profesional, junto con todo tipo de restaurantes que aceptan cheques-restaurantes (facilitados por las empresas a sus empleados). Nos aproximamos al tema de la restauración ya en 1992 y recurrimos a la misma fuente para comprobar las nuevas pautas.

Pues bien, la actual oferta de restauración en Madrid, parece cumplir con «la necesidad de comer es biológica, pero la comida rápida o la comida francesa es un deseo y consumir la misma no sólo satisface las necesidades biológicas sino más importante confirma el consumo basado en la autoestima» (Lanhman, L., 1992). Así el consumo de lugares y de formas nuevas, no sólo se puede observar a través del mayor número de restaurantes, sino de qué tipo de especialidades ofrecen, la novedad del país o la recuperación de la tradición como sucede con la franquicia «Cañas y Tapas» que recrea las tabernas en decoración, menú, o el uso de la sartén como plato.

Desde los años noventa, Madrid cuenta con un mayor número de restaurantes con especialización regional española, extranjeros, e incorporados a la «moda», por ejemplo si en 1992 contaba con 4 especializados en «nueva cocina», en 1996 eran 9; mientras que la oferta vegetariana ha añadido catorce establecimientos más en 1999, y ante el actual reconocimiento de los expertos en dietética de la bondad de la cocina mediterránea, abundan ahora los restaurantes que anuncian su incorporación a la misma, 55 en diciembre de 1999.

${ }^{8}$ Las cadenas de hamburguesas están bien presentes en nuestro espacio urbano, pero en la actualidad la incidencia se produce en la modalidad de restaurante, que estaba representada por los Foxter Hollywood, a los que se han unido en los últimos años: Artis Century, Henry J. Bean's, Fifties, Friday's, y los Ribs que según la ubicación tienen ambiente «estilo tradicional», «estilo diseño», o «estilo country USA». 
- A la variedad y mayor número de restaurantes de cocinas regionales españolas, se han unido otros cuya especialización es incluso de difícil comprensión, desde cocina «ecléctica», «creativa», «variada», «moderna», «imaginativa», «clásica-renovada», «tradicional-renovada», «original», «imaginativa-terrorífica», «mercado», «alta-cocina», o «afrodisíaca».

- Un rasgo significativo, es la ampliación y variedad de la oferta «mundial», y así de los 114 restaurantes de cocina extranjera en 1992 se ha pasado a 172 en 1996 y a 198 en enero del 2000, y se observa una pérdida del dominio europeo, y la incorporación de mayor número de países asiáticos. Singularmente se observa una «americanización» derivada del mayor numero de establecimientos, argentinos, mejicanos (con ellos hemos recuperado la palabra cantina, tan sólo presente en pequeñas estaciones de tren), norteamericanos, y la incorporación de otros países de ese continente, de este modo se continúa la intercolonización, ya que sí con la aportación de productos se amplió la dieta europea, actualmente se incorpora la «cultura» del «modo de hacer», se recrea el ambiente mediante la decoración, el servicio, y el lenguaje, coincidiendo con la estética postmodernista. Este hecho va unido a la «comida rápida», «adaptación a los niños» y a los «jóvenes»; su localización es aislada en espacios centrales y en los centros comerciales de los municipios de la corona metropolitana. Como ejemplo destacaríamos, el gusto «americano» que ya apareció con la instalación de la Base Militar (USA) en Torrejon de Ardoz y permanece el restaurante «Rancho Texano» (inaugurado en 1962) en la autovía; pero ahora «coloniza» el corazón de los negocios» como se demuestra en la Castellana y parece señalar el estandarte de la estatua de Colón: ahí esta América con su «Hard Rock», su «Chicago's» completados a escasa distancia por la tienda «Taste of America».

- Sin embargo, examinado los precios, no parece que quienes apuestan por lo «exótico» sean los que pueden pagar altos precios, ya que entre los 76 restaurantes de máximo precio en enero del año 2000 , sólo aparece un chino y un japonés. El dominio de la cocina española en estos precios es absoluto, pero la preponderancia anterior de la cocina vasca y la marisquería aparece mitigada por la «moda» actual mediterránea o de mercado. Al igual que estos precios han perdido su exclusividad vocacional por el espacio central, se acoplan en su localización a nuevas áreas de actividad para ser complemento del «ocio-negocio» y al vencimiento de la distancia, se puede comer en otros municipios en razón al 
prestigio del lugar (entorno, por ejemplo $\mathrm{N}-\mathrm{VI}$ ) de un edificio singular (Palacio Negralejo, en Mejorada del Campo), o de un cocinero de renombre (en Moralzarzal, municipio de 3.672 hab. en 1996 y con un restaurante en la guía Michelín).

La mayor «mundialización» se produce en categorías medias y mediobajas, accesibles a demandas más «masivas». Así su localización se produce en espacios centrales, pero no exclusivamente, ya que también se ubican en distritos periféricos y en menor medida en la corona metropolitana. Ocurre que precios y ubicaciones parecen ir unidos al gusto por consumo de lugares nuevos y novedades culinarias, a las que se unen música o actuaciones diversas ${ }^{9}$, a lo que Bailly, A. y Paelinck, J. (1990) denominaron «restaurante teatro», y a la más novedosa incorporación en algunos de venta de artículos diversos, productos y utensilios culinarios, libros, discos, cuadros y toda la decoración (como en Pele-Mele). A ello podemos añadir, la oferta de otros servicios, locales que han incorporado como atractivo el acceso a la red Internet (Ciberteca, Net Cafe) bien localizados en zonas de ocio del espacio central. A esta mundialización culinaria se ha unido la vivienda, como lugar de consumo de la misma, incorporando a la dieta productos de las estanterías de las grandes superficies o de las tiendas especializadas, o bien mediante los teleservicios, o mediante los catálogos (el de Eismann, por ejemplo, contiene páginas dedicadas a «Asia World» $\mathrm{y}$ «American Style»).

- Incorporación de mayor espacio de consumo para satisfacer las «necesidades de comida» desde los años noventa; en efecto progresivamente se anuncia en prensa «comer fuera de Madrid» (capital), fenómeno ligado en principio al fin de semana, y ahora incorporándose los municipios de la corona metropolitana para satisfacer a los residentes y a los atraídos por un entorno de menor densificación, añadiendo las periferias a las pautas de centralidad o una mayor difusión de los rasgos centrales a espacios más alejados, y el uso del automóvil. Hay una presencia en los centros comerciales que actúan como imanes y se han reforzado los ejes de carreteras, especialmente el de autovía de La Coruña, por su tradición, su «calidad» ambiental, sus connotaciones de estatus residencial, su atracción para la ubicación de centros comerciales y de empresas.

${ }^{9}$ A la cena con espectáculo, tradicionalmente flamenco, se han unido las salas rocieras, músicas sudamericanas, mariachis, actuaciones infantiles, proyecciones de videoclips, o la danza del vientre. 
- En definitiva «comer fuera de casa» ${ }^{10}$ parece un consumo extenso e intenso: a mayores capas sociales, a mayor oferta de restaurantes especializados, asociados y no relacionados específicamente con otras actividades, a proposiciones de enclaves variados.

\section{Los Cines}

Representa una expresión de tiempo de ocio ya tradicional, anunciada hace años en regresión frente a nuevas modalidades, y renacida tras su adaptación a las nuevas pautas de consumo entre las que podemos citar: desaparición de las salas que no se incorporan al fenómeno de oferta variada en el mismo punto y en cuyos locales se instalaron bingos, gimnasios etc.; éxito de las salas que dividen sus antes masivos aforos, en reducido número de butacas y simultaneidad de películas exhibidas; ampliación de la oferta horaria en la que hay que destacar los pases desde las 12 horas, en la tradicional franja de horario de comidas, hasta los más noctámbulos que pueden acudir a la 1,20 de la madrugada; y diversidad de lugares de proyección.

El espacio del cine se muestra muy selectivo, y el antes cine de «barrio», con películas de reestreno, sito en calles principales que actuaban como centro para esos barrios, se ha convertido en multisala o en la mayor parte de los casos su local se dedica a otras actividades. Pero se mantienen las áreas dentro del espacio central de espectáculo, restauración y compras, por ejemplo Gran Vía y Fuencarral (con marcada tendencia a la sustitución en multicines), es decir en áreas accesibles y complementadas con otras actividades. En los distritos periféricos se han ido cerrando cines, transformándose en multicines, y abriéndose las multisalas ubicadas en los centros comerciales. De la misma manera, en los municipios de las coronas metropolitanas ha «explosionado» la misma tipología y especialmente en los centros comerciales: en 1992, 9 centros comerciales en 8 municipios, presentaban una variada oferta en número de salas, desde los más modestos de dos o tres a los de ocho y nueve salas,

${ }^{10}$ La extrema rapidez de «comer por la calle o en la mesa de trabajo», no «aparece» pero Mark \& Spencer vende en su supermercado, cajitas individuales con ensaladas, otros «tentempiés» y proporciona cubiertos de plástico; y también se venden en establecimientos diversos «café» en vaso hermético de cartón que se calienta cuando lo abres y por lo tanto podremos dejar de ir a tomar café a la cafetería, e incluso de levantarnos a la máquina. 
en 1996 eran 17 C.C. en 15 municipios y en enero del 2000, 24 C.C. en 17 municipios, llegando el número de salas en el mismo punto a más de veinte. No es por lo tanto de extrañar que la prensa y las guías de ocio ofrezcan páginas dedicadas a «Cines de la periferia», «Cines Comunidad de Madrid» 0 «Salas de la Comunidad», ya que el amplio horario, los precios según sesiones y especiales (para niños, minusválidos, carnet joven, tarjetas de filiación), la seguridad del aparcamiento, reserva de entradas (vía teléfono, cajeros automáticos o internet), accesibilidad por las vías rapidas de comunicación, y la misma cartelera, junto con la complementariedad de tomar algo en el mismo lugar, parecen facilitar los desplazamientos entre municipios y distritos periféricos. Efectivamente para contemplar la «última película» ya no hay que ir al espacio central, ha desaparecido la división y ubicación de cines de estreno y de reestreno, con su diferente precio; ahora lo «último» está en variados puntos, parece más «accesible» (necesidad de vehículo) y llegar a mayor número de personas.

El cine ha renacido en espacios específicos, ha asumido el papel de locomotora antes reservado para los hipermercados en los centros comerciales, se asocia a otros usos del suelo antes específicos, estación de tren, parques, y recientemente se convierte en motor de atracción de enclaves de ocio, no inserto en C.C., pero no lejos de focos de consumo complementarios, en Madrid podemos citar: desde su presencia en la Estación de Chamartín; el Parque Tierno Galván; su acomodación en un espacio que ha cambiado de vocación (Méndez Álvaro); su ubicación en un «polígono», en Pozuelo de Alarcón, con nombre de «Ciudad de la Imagen», donde dormir se reduce a un hotel, las compras a un hipermercado, la actividad es intensa en relación con la denominación, en un complejo exclusivo «Kinépolis» de 25 salas que se complementan con bares y restaurantes; a su instalación en otro «polígono de consumo», de inmensas manzanas, donde se ubica desde un centro comercial con multicines a una pista de patinaje sobre hielo, y donde la Warner Lusomundo compite en Majadahonda, con su C.C. «Equinocio» más centrado en el ocio, «más de 2.600 localidades» y restauración que comercio; al reciente centro de ocio, con vocación de ciudad «Heron City» la «Megaciudad del Ocio y la Diversión» en Las Rozas, que inaugura un espacio interior al aire libre recreando la «plaza del pueblo», desde el cual se accede a las 24 salas de cine, a múltiples establecimientos para comer o beber algo, a salas de juegos, al gimnasio mejor su denominación «Health lands», y en la cual se recrea «lo espontáneo» de la plaza, con acróbatas, payasos, pero controlado 
ya que es un espacio privado, y donde los juegos de luz, agua y música forman parte del atractivo. Esta ciudad «Heron city» parece un «simulacro» de la suburbanización y aculturarización, ya que hace pensar en una -América, América - como intenta Sir Jack (Barnes, J. 1999) con su «Inglaterra, Inglaterra» en la isla de Wight. Pero el consumo parece llevar a más consumo, de bienes... y de servicios con caducidad más corta, mientras que el consumo de ocio ofrece «infinitas satisfacciones», por lo cual, las consultoras inmobiliarias apuestan por este mercado, el auge de los centros comerciales y de ocio, calificándolo de «producto estrella» en este momento.

\section{Conclusiones}

A través de las actividades seleccionadas, comerciales, restauración y cines, y de la exposición de nombres y publicidad, hemos tratado de aproximarnos al espacio y formas de consumo en Madrid, teniendo en cuenta la dimensión de la aglomeración, la ampliación de los enclaves para consumir, la diversificación de las ofertas, etc. Creemos que hemos dado una visión simplificada de esa variedad, pero con la pretensión de que sea expresiva de: la unión de actividades (comercio, restauración y ocio); la dificultad de acceder en el espacio urbano, una aglomeración, al uso y al conocimiento del mismo por parte de los residentes y del investigador; y la incorporación en la oferta de servicios a la mundialización y la masificación de los comportamientos. Los madrileños parecen consumir más, más diversificado, más especialidades, más novedades, más lo «último», más informados, más transnacionalizados, más rápidamente; y ejemplo todo lo anterior puede ser la moda de la «pashmina»: que ha sustituido a la bufanda, al chal, al pañuelo de cuello, que es distribuida por marcas y lugares exclusivos y por puestos ilegales en el suelo de las calles, llevada anudada por capas sociales muy altas y en el metro dependiendo de calidad-precio, con trajes de gala y de sport, por hombres y mujeres. Es de esperar que la inmersión en esta «cultura del consumo» sea en beneficio de los madrileños y de cuantos «Isidros» nos visiten ya que los nuevos espacios son muy competitivos y atractivos, en precios, ofertas e imagen, para los residentes en las autonomías vecinas que ven mejorada su accesibilidad con la red de carreteras y ferrocarriles, y su dificultad de entrar en la «ciudad», facilitada por las proximidad de los nuevos lugares 
de consumo a vías rápidas y el aparcamiento «seguro» que proporcionan esos «centros».

\section{BIBLIOGRAFÍA}

Alonso, J., ReBollo, A. y OuBiÑA, J. (2000): «Análisis de la demanda y estructura del consumo en España: consumidor español-consumidor europeo», en El consumo en España: un panorama general (Gimeno, J. A. coord.). Fundación Argentaria - Visor Dis., Madrid, pp. $79-140$.

ALONSO, L. E. y CONDE, F. (1994): Historia del Consumo en España: una aproximación a sus origenes y primer desarrollo. Debate, Madrid, $254 \mathrm{pp}$.

Atkins, P., Simmons, I. \& Roberts, B. (1998): People, land \& time. Arnold, London, 286 pp.

AYUNTAMIENTO DE MADRID. (1999): «Los derechos del consumidor en el municipio de Madrid». Encuesta Municipal de Consumo, 95 pp.

BaIlly, A. \& PAELINCK, J. (1990) «Restaurants, Espace, Temps», en Cahiers du Tourisme, série C, n. ${ }^{\circ}$ 137, Centre Des Hautes Etudes Touristiques, Aix-en Provence, 32 pp.

Ballesteros, C. (1999): La imagen de los hipermercados en la Comunidad Autónoma de Madrid, U. P. Comillas, $261 \mathrm{pp.}$

BARNES, J. (1999): Inglaterra, Inglaterra. Anagrama, Barcelona, 317pp.

Bustos, D. y GARCíA, E. (1990): «Dinámica de los Centros Comerciales y Grandes Superficies Comerciales en el Area Metropolitana de Madrid», en Primeras Jormadas de Geografía y Comercio: Grandes Superficies Comerciales, IGU y UCM, Madrid, pp. 190-204.

Bustos, D. y García, E. (1992): «El centro, ocio, negocio y cultura», en Atlas de la Ciudad de Madrid, Ideographis, Madrid, pp. 116-119 y 214-216.

CARRERAS, C. (1995): «Los nuevos espacios del consumo en Barcelona», Revista de Geografía, Universitat de Barcelona, vol. XXIX, pp. 69-77.

CASARES, J. (1999): «La actividad comercial», en Estructura Económica de Madrid (García Delgado, J. L. Dir.), pp. 477-502.

CCCE (1997) «La Económica Regional en cifras 1996». Gráficas Rofer, Madrid, 103 pp.

FERREIRA, R. (1996): Centres Commerciaux: $̂$ les urbaines de la post-modernité. E. L'Harmattan, París, $141 \mathrm{pp}$.

EsTÉBANEZ, J. (1994): «Reestructuración económica, cambio tecnología e influencia del espacio. El ejemplo de Madrid», en Madrid en el contexto de lo hispánico desde la época de los descubrimientos. UCM, Madrid, tomo I, pp.89-112.

Eurostad (1994): El Comercio al por menor en el Mercado Único Europeo, CE, Luxemburgo.

GARCÍA DELGADO, J. L. (1999): «Factores determinantes de la formación de la estructura económica de Madrid» en Estructura Económica de Madrid, Civitas, Madrid pp.3-20.

GARCíA, N. (1995): Consumidores y ciudadanos. Conflictos multiculturales de la globalización, Grijalbo, México, $198 \mathrm{pp}$.

GoulA, J. (1997): «Por fin despierta el consumo», Economics, n. ${ }^{\circ}$ 110, Barcelona, pp. 8-9.

HEALEY \& BAKER (1995): Main streets across the world 1995. London, $27 \mathrm{pp}$.

JACKSON, P. and TAYLOR, J. (1996): «Geography and the cultural politics of advertising», Progress in Human Geography 20, 3, pp. 356-371.

LANGMAN, L. (1992): «Neon gages: shopping for subjectivity», en Lifestyle Shopping. The Subject of Consumption. (E. Shields, R.), Routledge, London, 239 pp.

McInTosh, A. (1997): Towns and Cities. E \& FN Spon, U.K. 166 pp.

MelLe, M. (1999): «El comercio interior en la Comunidad Autónoma de Madrid: actualidad, caracterización y tendencias», Papeles de Economía Española, n. ${ }^{\circ} 18$, Madrid, pp. 316332.

$$
-101-
$$


MÉNDEZ, R. y ONDÁTEGUI, J. (1999): «La estructura territorial del sistema productivo», en Estructura Económica de Madrid (García Delgado, J. L. Dir.), pp.131-166.

MetTon, A. (1989): Le commerce et la ville en banlieue parisienne. Metton, Courbevoie.

PADOVANI, E. (1999): «Changer pour rester dans l'espace: le cas du «télémarketing» ou»marketing» direct», en The Globalization of Consumption and Retail Places (Barata, T. ed.), GECIC, Lisboa, pp. 263-273.

Precedo, A. (1996): Ciudad y desarrollo urbano. E. Síntesis, Madrid, 247 pp.

SHIELDS, R. (1992): «The individual, consumption cultures and the fate of community», en Lifestyle Shopping: the subject of consumption. Routledge, London, $239 \mathrm{pp}$.

SoumAGNE, J. (1999): «La diffusion spatiale des sociétés commerciales etrangeres en France» en The Globalization of Consumption and Retail Spaces (Barata, T. ed.), GECIC, Lisboa, pp. 99-121.

ThrifT, N. and GlEnNie, P. (1993): «Historical Geographies of Urban Life and Modern Consumption», en Selling Places. The City as Cultural Capital, Past and Present, (E. Kearns, G. and Philo, C.). Pergamon Press, Oxford, pp. 33-48.

THRIFT, N. and OLDS, K. (1996): «Refiguring the economic in economic geography», Progress in Human Geography 20, 3, pp.311-337.

YAGÜE, M. ${ }^{\mathrm{a}}$ J. (1998): «Madrid como ciudad comercial: implantación y extensión de las grandes superficies comerciales». Economistas, n. ${ }^{\circ}$ 79, Madrid, pp. 220-234.

RESUMEN: Se presentan las nuevas formas y lugares de consumo en la aglomeración urbana madrileña, la explosión de lugares, formas, la concentración, la masificación, la búsqueda de la singularidad producto del individualismo de fines del siglo $\mathrm{xx}$. A traves de tres variables seleccionadas: comercio, restauración y cine, teniendo en cuenta su ubicación y especialización en el entramado urbano.

PALABRAS Clave: Consumo, comercio, restauración, cine, Madrid.

ABSTRACT: This work discusses about the news ways and places of consumption in the urban agglomeration of Madrid, as well the explosion of sites and shapes, the commercial massification, and on the opposite direction, try to selfdistinguish according to the typical individualism in the end of the century. Three variable are been selected: retail, restaurants and cinemas, and the special attention to its location and specialization in the townscape.

KEY WORDS: consumption, retailer, restaurants, cinemas, Madrid. 